\title{
Growth functions and their application in animal science
}

\author{
J France ${ }^{1}$, J Dijkstra ${ }^{1}$, MS Dhanoa ${ }^{2}$ \\ 1 Institute of Grassland and Environmental Research, North Wyke Research Station, Okehampton, \\ Devon EX20 2S; 2 Institute of Grassland and Environmental Research, Plas Gogerddan, Aberystwyth. \\ Dyfed SY23 3EB, United Kingdom
}

\begin{abstract}
Summary - Brief consideration is given to some simple growth functions commonly applied in animal science and other fields of biology, namely the exponential (with abrupt cut-off), monomolecular, logistic and Gompertz. Consideration is then given to two nested growth functions, the Richards and a new function which takes the form of a generalised Mitscherlich equation and is capable of describing both diminishing returns and sigmoidal patterns of growth. The two functions are compared using sets of growth data on cows, goats, pigs, dogs and geese.
\end{abstract}

\section{Introduction}

Growth functions provide a mathematical summary of time course data on the growth of an organism or part of an organism. The term growth function is generally used to denote an analytical function which can be written down as a single equation. Thus, a general growth function connecting weight $W$ to time $t$ is

$$
W=f(t)
$$

where $f$ is some functional relationship.

In animal science, growth functions have been used since the early years of this century. Key papers in the development of this area are those by Robertson $(1908,1923)$ on the logistic equation, Spillman and Lang (1924) the monomolecular, Winsor (1932) the Gompertz, and von Bertalanffy (1957) on a forerunner of the Richards equation. A useful monograph on the theory of feeding and growth in animals is that by Parks (1982), and the classic reference book on bioenergetics and growth, which is well worth browsing, is that by Brody (1945).

The use of growth functions is largely empirical. The form of the function $f$ will sometimes be chosen by simply looking at the data and making an informed guess. However, it is preferable to select or construct a function that has some biological plausibility, and whose parameters may be meaningful, i.e. they may characterize some underlying physiological or biochemical mechanism or constraint. In this paper, therefore, consideration is only given to functions that can be derived from a simple mechanistic model, by integration of a differential equation of the form rate is a function of state (Dijkstra and France,
1995; France and Theodorou, 1995). This allows meaning to be associated with the parameters of the W:t equation (equation 1 ).

\section{Simple growth functions}

In this section we give brief consideration to some commonly-applied simple growth functions, namely the exponential (with abrupt cut-off), monomolecular, logistic and Gompertz. A more complete account is given in France and Thornley (1984).

\section{Exponential with abrupt cut-off}

The underlying assumptions are the quantity of growth machinery is proportional to the weight of the organism $W$, growth machinery operates at maximum effectiveness so long as there is substrate available, growth is irreversible and stops immediately the substrate is exhausted. The rate:state equation is therefore

$$
\mathrm{d} W / \mathrm{d} t=\mu W
$$

where $\mu$ is the specific (or relative) growth rate. The parameter $\mu$ depends not only on the proportion of $W$ which constitutes growth machinery but also on the efficiency or speed with which this machinery can operate. Integration of the rate:state equation (equation 2) with the initial condition $W=W_{0}$ at $t=0$ yields

$$
\begin{aligned}
W & =W_{0} \mathrm{e}^{\mu t}, \quad 0 \leq t<t_{f} \\
& =W_{f}, \quad t \geq t_{f}
\end{aligned}
$$

where $W_{f}$ is the final weight reached at time $t_{f}$ when substrate becomes exhausted. Equation 
(3) is illustrated in figure 1 . The growth rate increases continually (until $t_{f}$ ) and there is no point of inflexion, which can be seen from the second differential

$$
\mathrm{d}^{2} W / \mathrm{d} t^{2}=\mu^{2} W_{0} \mathrm{e}^{\mu t}
$$

\section{Monomolecular}

Assumptions are the quantity of growth machinery is constant and independent of organism weight $W$, this machinery works at a rate proportional to substrate level $S$, and growth is irreversible. The monomolecular describes the progress of a simple irreversible first-order chemical reaction. The rate:state equation is

$$
\mathrm{d} W / \mathrm{d} t=\mu S
$$

where $\mu$ is a constant. Writing $S$ as $W_{f}-W$, where $W_{f}$ is final (mature) weight, equation (5) becomes

$$
\mathrm{d} W / \mathrm{d} t=\mu\left(W_{f}-W\right)
$$

Integrating of this rate:state equation with $W=W_{0}$ at $t=0$ gives

$$
W=W_{f}-\left(W_{f}-W_{0}\right) e^{-\mu t}
$$

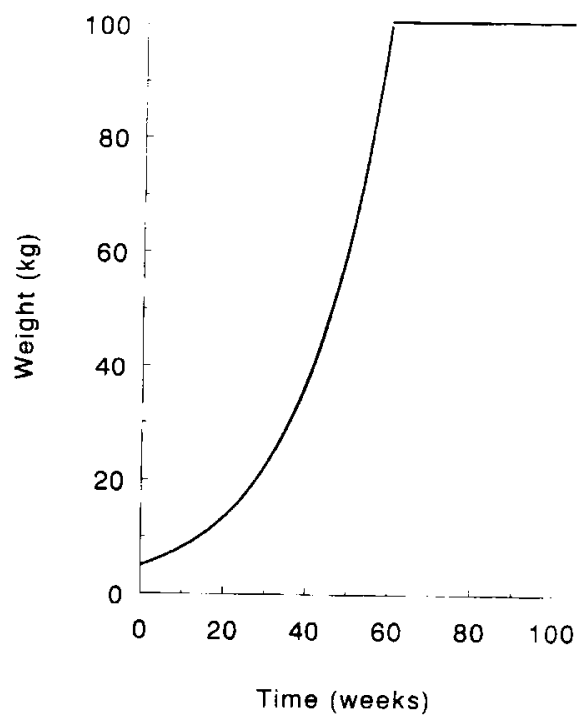

Figure 1. Exponential growth with abrupt cutoff. The curve shows equation (3) with $W_{0}=5$, $W_{f}=100, \mu=0.05$ and $t_{f}=60$.
Equation (7) is illustrated in figure 2. The growth rate decreases continually and there is no point of inflexion. This can be seen from the second differential

$$
\mathrm{d}^{2} W / \mathrm{d} t^{2}=-\left(W_{f}-W_{0}\right) \mu^{2} \mathrm{e}^{-\mu t}
$$

which is zero for only $t \rightarrow \infty$ or $W \rightarrow W_{f}$.

The monomolecular was developed by Spillman and co-workers at the USDA as a form of the law of diminishing returns to describe the live weight of an animal as a function of its food consumption (Spillman and Lang, 1924). Brody (1945) used the monomolecular in conjunction with the exponential to account for S-shaped growth patterns over time. He describes growth as "self accelerating" (exponential) before and "self inhibiting" (monomolecular) after time t', the age of puberty. Thus the Brody equation describes sigmoidal behaviour but with a discontinuity at the point of inflexion $t=t$ ' reflecting (according to Brody) the switch from being vegetative to being procreative.

\section{Logistic}

The logistic describes smooth sigmoidal behaviour with no discontinuities. The

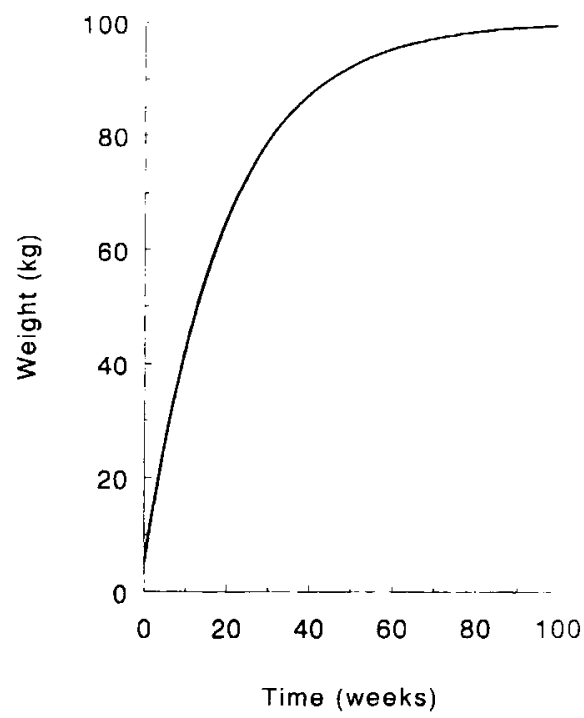

Figure 2. Monomolecular growth. The curve shows equation (7) with $W_{0}=5, W_{\mathrm{f}}=100$ and $\mu=0.05$. 
underlying assumptions are the quantity of growth machinery is proportional to organism weight $\mathrm{W}$, this works at a rate proportional to the amount of substrate $S$, and growth is irreversible. The rate:state equation is

$$
\mathrm{d} W / \mathrm{d} t=k W S
$$

Writing $k$ as $\mu / W_{f}$ and $S$ again as $W_{f}-W$, equation (9) becomes

$$
\mathrm{d} W / \mathrm{d} t=\mu W\left(1-W / W_{f}\right)
$$

With the initial condition $W=W_{0}$ at $t=0$, this rate:state equation can now be integrated to give the logistic

$$
W=W_{0} W_{f} /\left[W_{0}+\left(W_{f}-W_{0}\right) e^{-\mu t}\right]
$$

Equation (11) is illustrated in figure 3 . The single point of inflexion occurs when the organism reaches half its final weight, i.e. $W=W_{f} / 2$. This can be seen from the differential of equation (10)

$$
\mathrm{d}^{2} W / \mathrm{d} t^{2}=\mu\left(1-2 W / W_{f}\right) \mathrm{d} W / \mathrm{d} t
$$

The logistic was first derived by Robertson (1908) in studying autocatalysis and was subsequently introduced into animal science by him (Robertson, 1924).

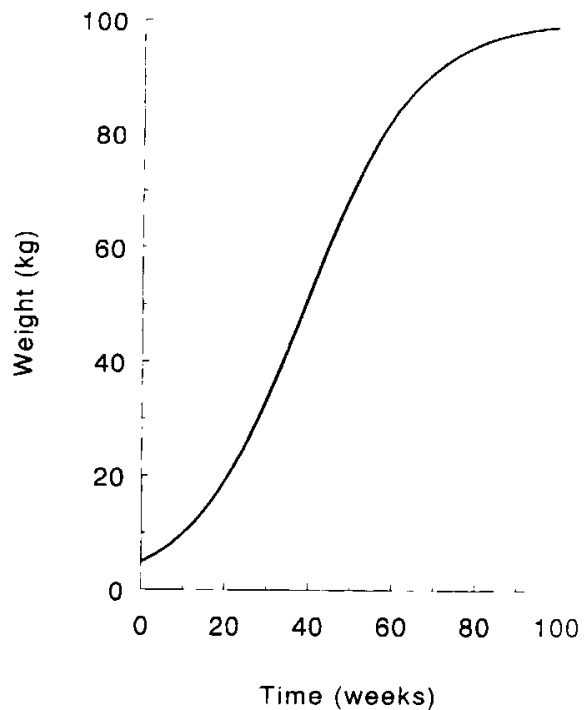

Figure 3. Logisitc growth. The curve shows equation (11) with $W_{0}=5, W_{f}=100$ and $\mu=0.075$

\section{Gompertz}

The Gompertz, like the logistic, describes smooth sigmoidal behaviour with no discontinuities. It is assumed that the quantity of growth machinery is proportional to organism weight $W$ with proportionality $\mu$, the effectiveness of the growth machinery decays with time according to first-order kinetics, substrate is nonlimiting, and growth is irreversible. Formalising these assumptions gives

$$
\begin{aligned}
\mathrm{d} W / \mathrm{d} t & =\mu W \\
\text { and } & \\
\mathrm{d} \mu / \mathrm{d} t & =-D \mu
\end{aligned}
$$

where $\mu$ is the specific growth rate and $D$ a decay parameter. Integrating equation (14) with the initial condition that $\mu=\mu_{0}$ at $t=0$ and substituting in (13) yields

$$
\mathrm{d} W / \mathrm{d} t=\mu_{0} W \mathrm{e}^{-D t}
$$

Integrating (15) with $W=W_{0}$ at $t=0$ then gives the Gompertz

$$
\begin{aligned}
& \ln \left(W / W_{0}\right)=\mu_{0}\left(1-\mathrm{e}^{-D t}\right) / D \\
& \text { i.e. } W=W_{0} \exp \left[\mu_{0}\left(1-\mathrm{e}^{-D t}\right) / D\right]
\end{aligned}
$$

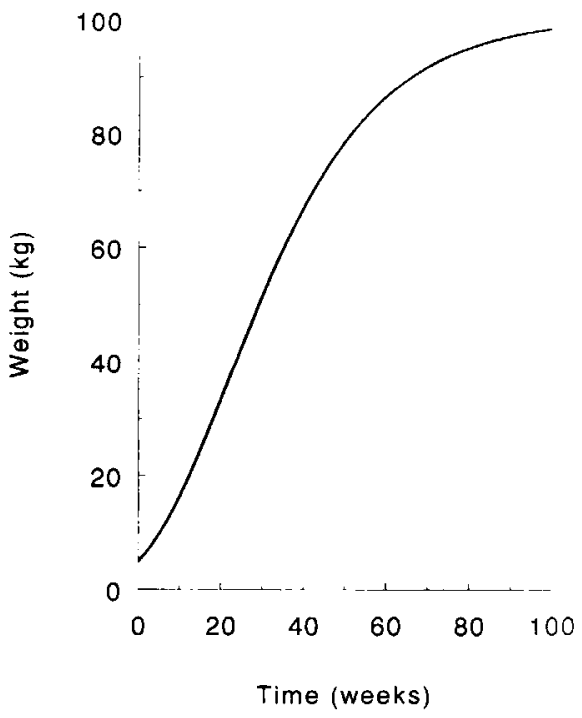

Figure 4. Gompertz growth. The curve shows equation (17) with $W_{0}=5, \mu=0.15$ and $D=0.05$. 
As $t \rightarrow \infty$, an asymptote at $W=W_{f}$ is approached, where

$$
W_{f}=W_{0} \exp \left(\mu_{0} / D\right)
$$

Equation (15) can be put in the rate:state form by substituting for $\mathrm{e}^{-D t}$ from (16), giving

$$
\mathrm{d} W / \mathrm{d} t=\mu_{0} W\left[1-\left(D / \mu_{0}\right) \ln \left(W / W_{0}\right)\right]
$$

The Gompertz (equation 17) is depicted in figure 4 . The single point of inflexion occurs when the organism reaches $1 / \mathrm{e}$ times its final weight, i.e. $W=W_{f} /$ e. This can be seen by differentiating equation (15)

$$
\mathrm{d}^{2} W / \mathrm{d} t^{2}=\mu_{0} \mathrm{e}^{-D t} W\left(\mu_{0} \mathrm{e}^{-D t}-D\right)
$$

and equating to zero, giving an inflexion point at $t=D^{-1} \ln \left(\mu_{0} / D\right)$ i.e. $W=W_{0} \exp \left(\mu_{0} / D-1\right)$.

Winsor (1932) was one of the first to propose the use of the Gompertz in animal science. It gives faster early growth but a slower approach to an asymptote than the logistic. These are arguably the two most commonly used growth functions.

\section{Nested growth functions}

In this section two nested growth functions, the Richards and a new function, are briefly

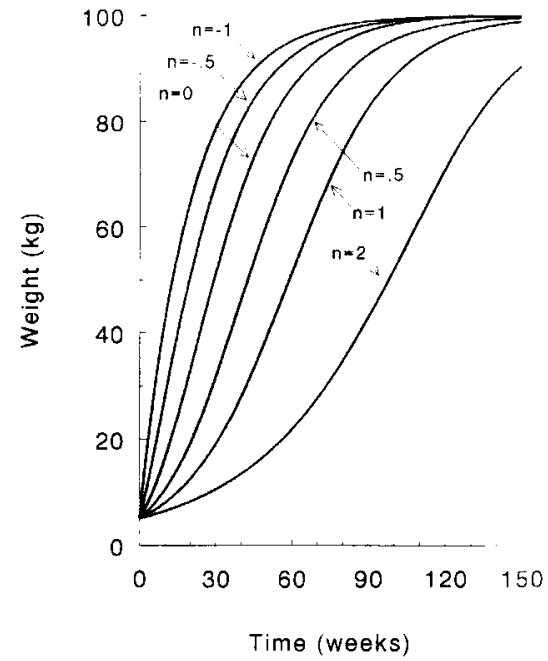

Figure 5. Richards growth. The curves show equation (22) with $W_{0}=5, W_{f}=100, \mu=0.05$ and for six values of $n$. $n=-1$ gives the monomolecular, $n=0$ the Gompertz and $n=1$ the logistic.

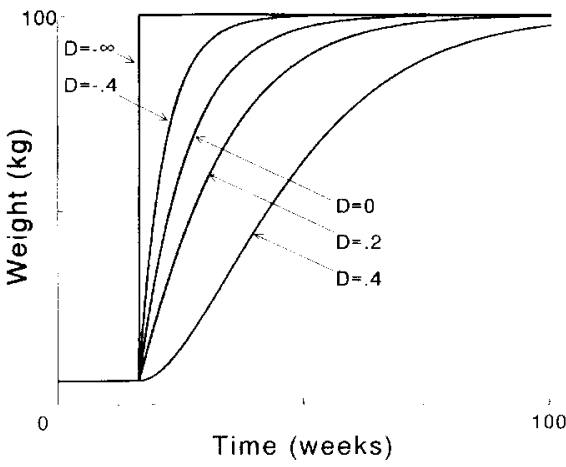

Figure 6. New function growth. The curve show equation (26) with $W_{0}=5, W_{f}=100$, $\mu_{\infty}=0.1, T=16$ and for five values of $D$.

considered. They encompass the previously described monomolecular, logistic and Gompertz, and have a generality which is often useful.

\section{Richards}

This is largely an empirical construct which owes its generality to an additional parameter $n$. The rate:state equation is

$$
\mathrm{d} W / \mathrm{d} t=\mu W\left(W_{f} n-W^{n}\right) /\left(n W_{f}^{n}\right)
$$

where $\mu, W_{f}$ and $n$ are constant. $\mu$ and $W_{f}$ are positive and $n \geq-1$, since $n<-1$ is nonphysiological giving infinite growth rate as $W \rightarrow 0$. With $n=-1$, equation (21) gives the monomolecular (equation 6 ). With $n=+1$, it gives the logistic (equation 10). When $n=0$, the Gompertz can be derived (see France and Thornley, 1984, p85). Using the initial condition $W=W_{0}$ at $t=0$, equation (21) can be integrated to give

$$
W=W_{0} W_{f} /\left[W_{0}^{n}+\left(W_{f}^{n}-W_{0}^{n}\right) \mathrm{e}^{-\mu t}\right]^{1 / n}
$$

Equation (22) is illustrated in figure 5 for different values of $n$. The point of inflexion is able to occur at any fraction of the mature weight as $n$ varies over the range $-1 \leq n<\infty$. This can be seen from the second differential

$$
\begin{aligned}
& \mathrm{d}^{2} W / \mathrm{d} t^{2}=\left[W_{f}^{n}-(n+1) W^{n}\right]\left[\mu /\left(n W_{f}^{n}\right)\right] \\
& \mathrm{d} W / \mathrm{d} t
\end{aligned}
$$

which gives a point of inflexion at:

$$
W=W_{f} /(n+1)^{1 / n} \text {. }
$$

The function was originally developed to study plants (Richards, 1959) from an equation 
by Von Bertalanffy (1957) to describe the growth of animals. A more complete account than the one presented here can be found in France and Thornley (1984).

\section{A new function}

The rate:state equation for this new function is the same as for the monomolecular (equation 6 ) but with the specific rate parameter $\mu$ allowed to vary with time as with the Gompertz. Growth rate is therefore defined by

$$
\mathrm{d} W / \mathrm{d} t=\mu\left(W_{f}-W\right)
$$

The time dependent rate parameter $\mu$ is given by

$$
\begin{aligned}
\mu & =0, \quad t<T \\
& =\mu_{\infty}-D / \sqrt{ } t, \quad t \geq T
\end{aligned}
$$

$T(\geq 0)$ is a time delay (before which $\mu=0$ ), $\mu_{\infty}$ $(\geq 0)$ is the value of $\mu$ at infinite time, and $D$ is a constant. Since $\mu \geq 0$, the maximum value of $D=\mu_{\infty} \gamma T$. A range of values of $D$ is considered in figure 6 which shows the full range of behaviour of the growth function.

It is possible to provide a tentative interpretation of equations (24) and (25). Many diffusion processes exhibit a square root time dependence. For instance, the distance diffused in time $t$ is $\sqrt{ }(4 D t)$ where $D$ is the diffusion constant. The diffusion of a growth activator into a growth-producing region, after a lag time, could lead to an activation equation similar to (25). The term $W_{f}-W$ in equation (24) measures the amount of substrate available for growth.

Substituting for $\mu$ in equation (24) using (25) and integrating with the initial condition $W=W_{0}$ at $t=0$ gives

$$
\begin{gathered}
W=W_{0}, \quad t<T \\
=W_{f}-\left(W_{t}-W_{0}\right) \exp \left[-\mu_{\infty}(t-T)+2 D(\sqrt{t}-\right. \\
\sqrt{T})], t \geq T
\end{gathered}
$$

Differentiating twice with respect to $t$ gives

$$
\begin{gathered}
\mathrm{d} W / \mathrm{d} t=0, \quad t<T \\
=\left(W_{f}-W_{0}\right)\left(\mu_{\infty}-D / \sqrt{ } t\right) \exp \left[-\mu_{\infty}(t-T)+2 D\right. \\
(\sqrt{t}-\sqrt{ } T)], \quad t \geq T
\end{gathered}
$$

and

$$
\begin{gathered}
\mathrm{d}^{2} W / \mathrm{d} t^{2}=0, \quad t<T \\
=\left(W_{f}-W_{0}\right)\left[D /\left(2 t^{3 / 2}\right)-\left(\mu_{\infty}-D / \sqrt{ } t\right)^{2}\right] \exp \\
{\left[-\mu_{\infty}(t-T)+2 D(\sqrt{ } t-\sqrt{ } T)\right], \quad t \geq T}
\end{gathered}
$$

Expanding, simplifying and equating to zero the term in square brackets containing $t^{3 / 2}$ in equation (28b), a point of inflexion may exist provided $D$ is positive if the cubic

$$
\mu_{\infty}^{2} t^{3 / 2}-2 \mu_{\infty} D t+D^{2} t^{1 / 2}-D / 2=0
$$

has a real root, $T \leq t<\infty$. Such a root will only exist if

$$
D /\left(2 T^{3 / 2}\right) \geq\left(\mu_{\infty}-D / N T\right)^{2}
$$

This can be seen graphically since the left side is a decreasing function of $T$ and the right side an increasing function of $T$. This leads to

$$
\sqrt{ } D \geq 0.5\left\{\sqrt{ }\left[1 /(2 \sqrt{ } T)+4 \mu_{\infty} \sqrt{ } T\right]-1 /\left(\sqrt{ } 2 T^{1 / 4}\right)\right\}
$$

which, for the parameters values used in figure 6 , gives $D \geq 0.23$ for a point of inflexion. Thus in figure $6,-\infty<D<0.23$ gives non-sigmoidal growth, whereas $0.23 \leq D \leq 0.4$ gives sigmoidal growth. A more detailed description of this growth function is given by France et al (in press).

\section{Comparison of nested functions}

In this section the new function and the Richards are compared briefly by fitting them to five sets of data on the growth of Guernsey cows, Saanen goats, Dutch Landrace boars, Great Dane dogs and Grey Lag geese. Both functions were fitted by $(A)$ varying (i.e. estimating) and (B) fixing (i.e. prescribing) the initial weight parameter $W_{0}$. The fits obtained from the Richards allowing $W_{0}$ to vary and from the new function fixing $W_{0}$ are used as the basis of the comparison because the same number of parameters had to be estimated in each case.

The parameter estimates and goodness-offit statistics are all given (to three significant figures) in tables I and II. The new function gives a better fit than the Richards to the data on Guernsey cows (RSS of 2410 v. 2800) and on Dutch Landrace boars (339 vs no convergence), but a poorer fit to the data on Great Dane dogs (70.2 vs 60.1 ). Similar fits were obtained to the data on Saanen goats and on Grey Lag geese. Fitting the extra parameter (i.e. $W_{0}$ ) improved the fit of the new function in the cases of the Guernsey cows, Dutch Landrace boars and Great Dane dogs (see table II). Fixing the parameter $W_{0}$ worsened the fit of the Richards function in the cases of the Guernsey cows, Great Dane dogs and Grey Lag geese (table I). It is interesting 


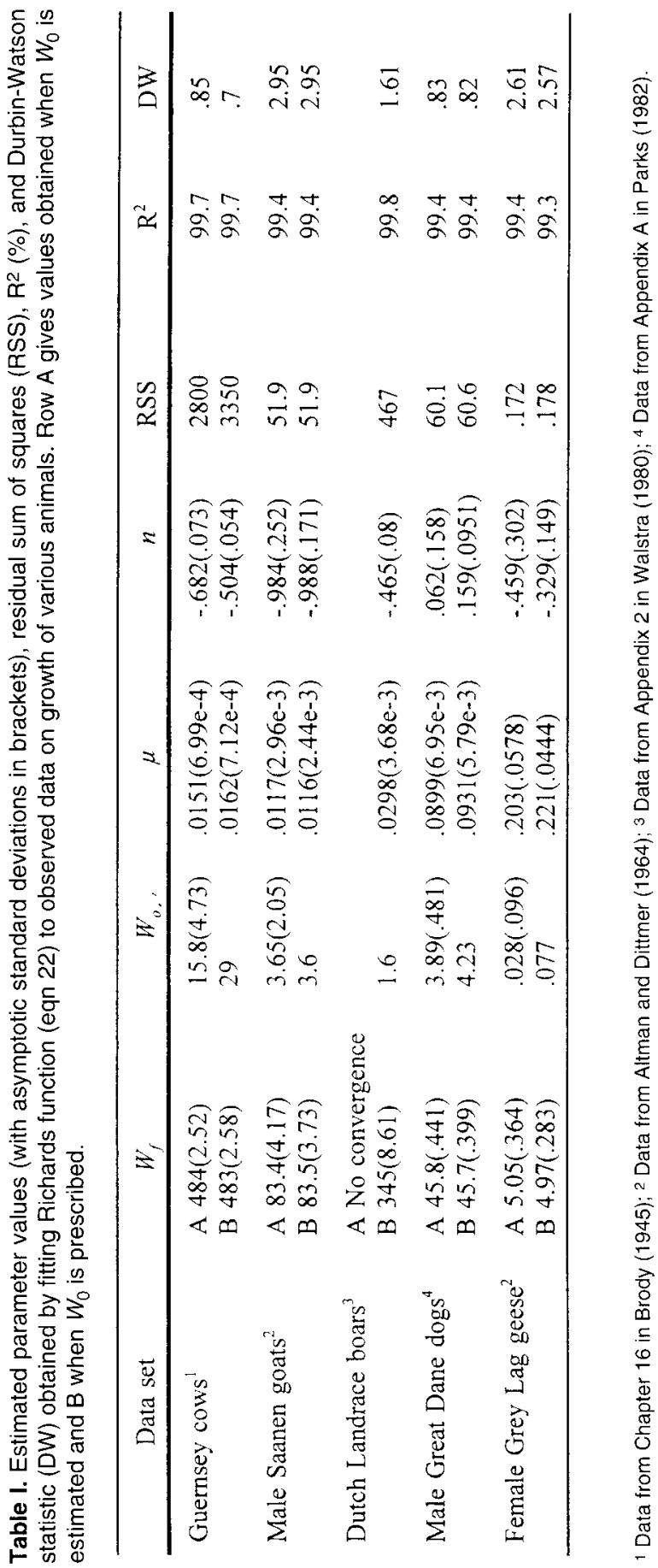




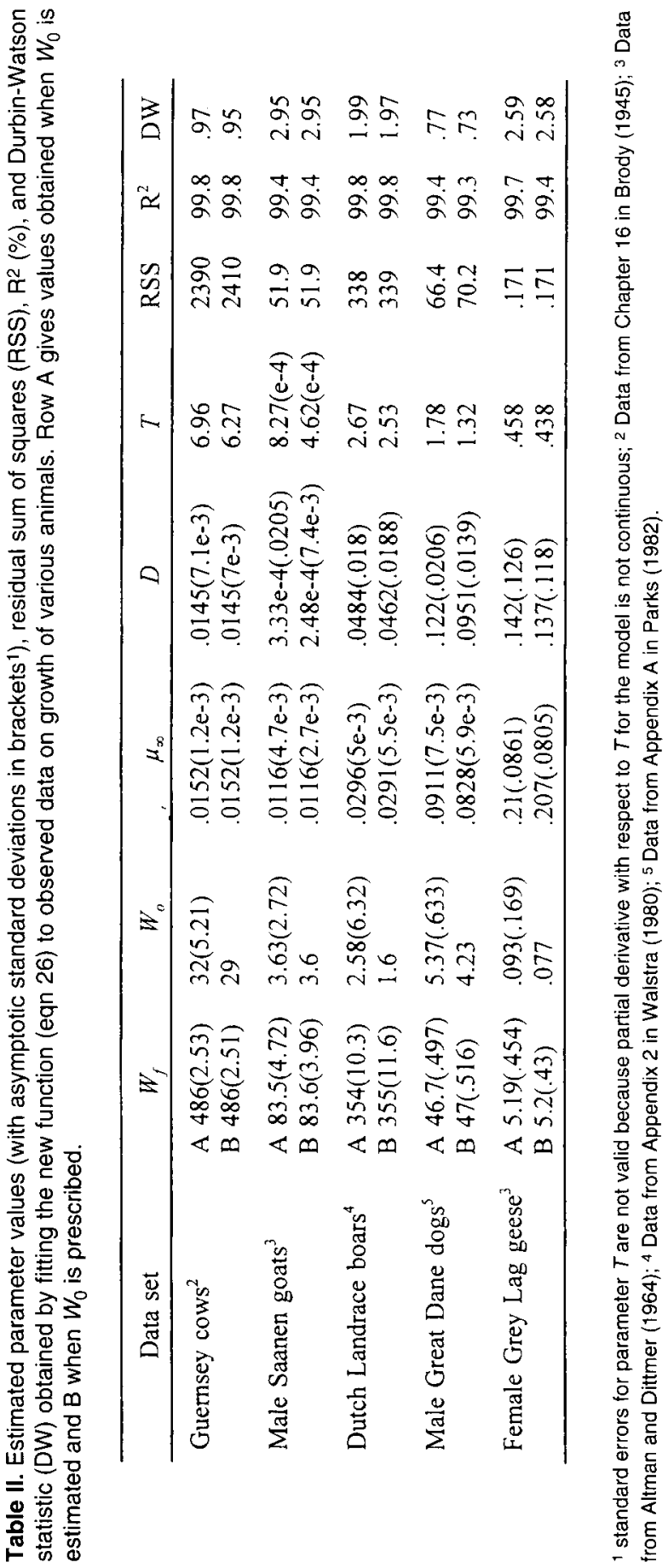




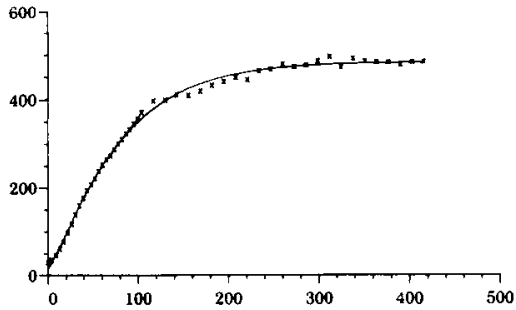

7.1 Guernsey cows (Richards)

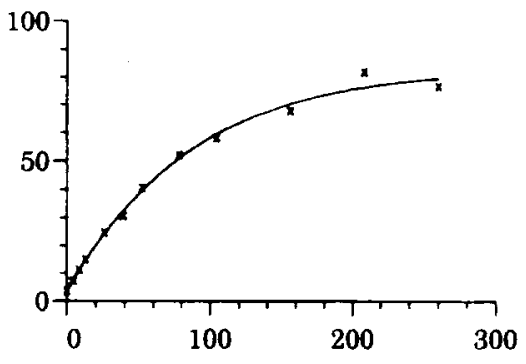

7.3 Male Saanen goats (Richards)

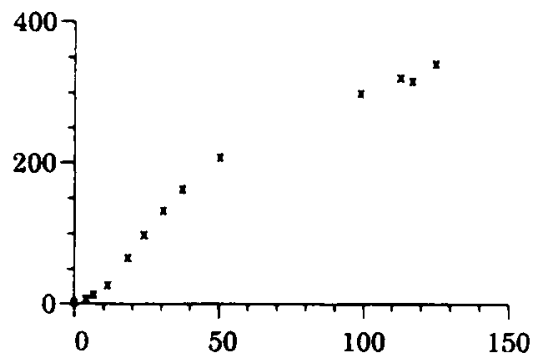

7.5 Dutch Landrace boards (Richards)

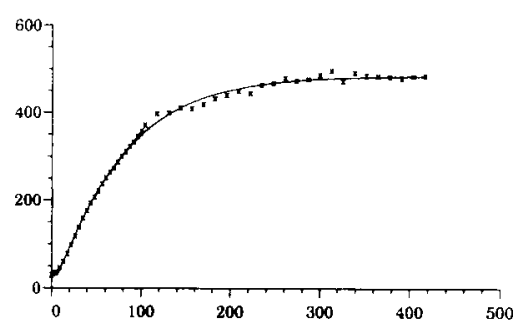

7.2 Guernsey cows (new function)

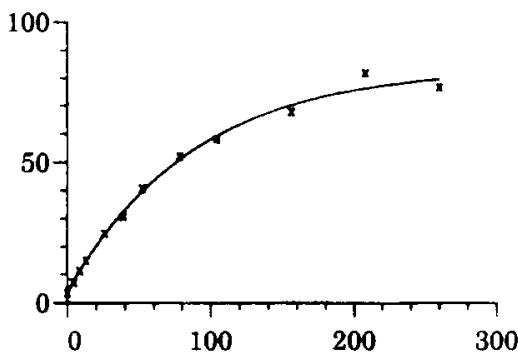

7.4 Male Saanen goats (new function)

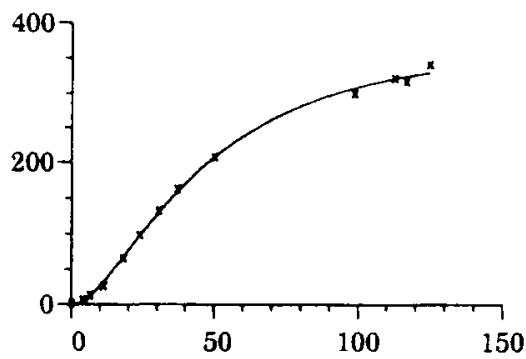

7.6 Dutch Landrace boards (new function)

Figure 7. Comparison of nested functions. The curves show the Richards and the new function (with $W_{0}$ prescribed) fitted to five sets of growth data. The vertical axes represent weight $(\mathrm{kg})$ and the horizontal axes are time (weeks). Continued on page 173 


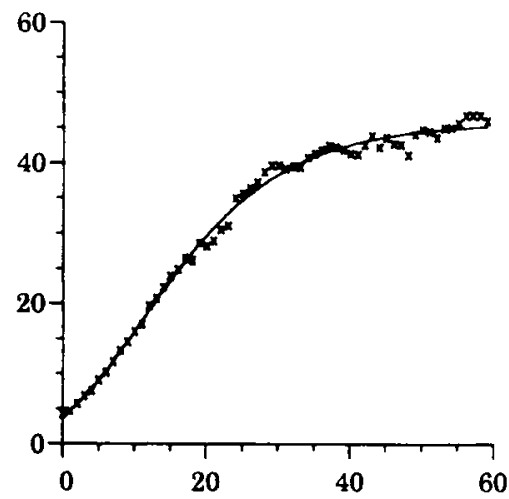

7.7 Male Great Dane dogs (Richards)

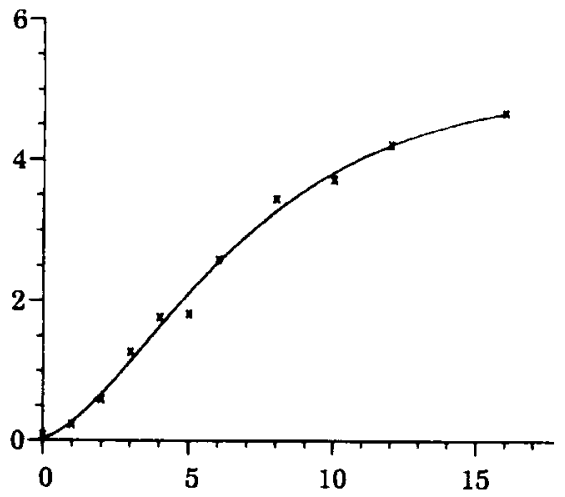

7.9 female Grey Lag geese (Richards)

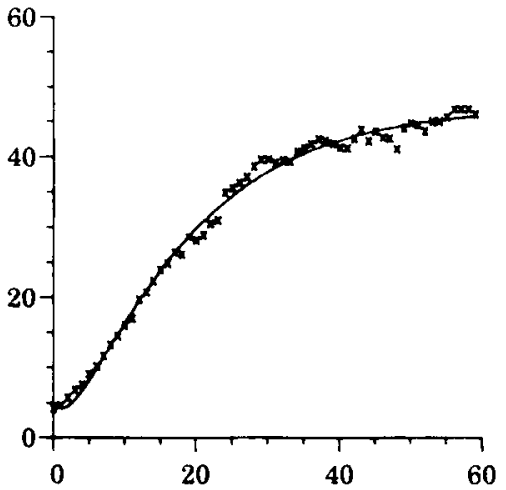

7.8 Male Great Dane dogs (new function)

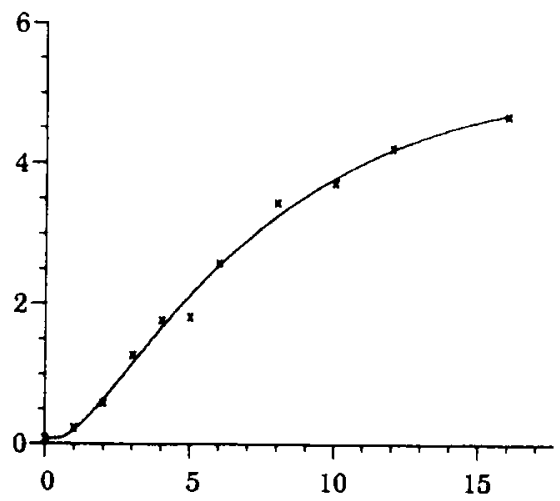

7.10 Female Grey Lag geese (new function)

Figure 7. see page 172

to note that the values obtained for the rate constants $\mu$ (Richards, table I) and $\mu_{\infty}$ (new function, table II) are similar for a given data set. The four-parameter fits of the two functions are illustrated for each data set in figure 7 .

In conclusion, the new function is capable of describing a range of diminishing returns and sigmoidal growth patterns. It has the advantages over the Richards of being able to describe a variety of possibilities, in addition to the monomolecular, when there is no point of inflexion and of having a more mechanistic derivation.

\section{Literature cited}

Altman PL, Dittmer DS (1964) Biology data book. Federation of American Societies for Experimental Biology, Washington DC

Brody S (1945) Bioenergetics and Growth. Rheinhold Publishing, New York

Dijkstra J, France J (1995) Modelling and methodology in animal science. In: Proceedings of the 4th International Workshop on Modelling Nutrient Utilisation in Farm Animals, (A Danfaer. P Lescoat, eds) October 3-5 1994, Research Centre Foulum, Denmark. National Institute of Animal Science, Foulum, 9-15 
France J, Thornley JHM (1984) Mathematical models in Agriculture, Butterworths, London, $335 \mathrm{p}$

France J, Theodorou MK (1995) Differential and integral equations and their application in quantifying the fungal population in the rumen. In: Anaerobic Fungi - Biology, Ecology and Function, (DO Mountfort, CG Orpin, eds) Marcel Dekker, Inc., New York, 257-270

France J, Dijkstra J, Thornley JHM, Dhanoa MS (1997) A simple but general growth function. Growth Dev Aging (submitted)

Parks JR (1982) A Theory of feeding and growth of animals. Springer-Verlag, Berlin

Richards FJ (1959) A flexible growth function for empirical use. J Exp Bot 10, 290-300
Robertson TB (1908) On the normal rate of growth of an individual and its biochemical significance. Arch Entwicklungsmech Org 25, 581-614

Robertson TB (1923) The chemical basis of growth and senescence. J.B. Lippincott Company, Philadelphia.

Spillman WJ, Lang E (1924) The law of diminishing increment. World, Yonkers.

Von Bertalanffy $L$ (1957) Quantitative laws for metabolism and growth. Q Rev Biol 32, 217-231.

Walstra P (1980) Growth and carcass composition from birth to maturity in relation to feeding level and sex in Dutch Landrace pigs, PhD Thesis, The Agricultural University, Wageningen, $206 p$

Winsor CP (1932) The Gompertz curve as a growth curve. Proc Nat Acad Sci, India 18, 1-8. 